\title{
Protection against the Omicron and Subsequent Coronavirus Variants: Medical-grade Masking, Third Dose Vaccination, Updating Vaccines, and Pursuing Universal Vaccine
}

\author{
Ahmad Shamabadi ${ }^{1,2}$ and Shahin Akhondzadeh ${ }^{2 *}$ \\ 1. School of Medicine, Tehran University of Medical Sciences, Tehran, Iran \\ 2. Psychiatric Research Center, Roozbeh Psychiatric Hospital, Tehran University of Medical Sciences, Tehran, Iran
}

^ Corresponding author: Shahin Akhondzadeh, Pharm.D., Ph.D., FBPhS, Psychiatric Research Center, Roozbeh Psychiatric
Hospital, Tehran University of Medical Sciences, Tehran, Iran
Tel: +982155412222 , Fax: +982155419113
Email: s.akhond@neda.net
Received: 15 Dec 2021

Accepted: 28 Dec 2021

The novel Omicron SARS-CoV-2 variant, also known as B.1.1.529, was first identified in South Africa in November 2021 and has now been reported in more than sixty countries and has become the dominant variant in the United States ${ }^{1,2}$. The Omicron's Ro could be as high as 10, while for the Delta, it was under seven. Also, the WHO called the risk "very high" 1 . The spread of this variant and the possibility of peaks resulting from it have raised concerns in many countries. Herein, the solutions are briefly introduced and discussed.

\section{Medical-grade masking up}

Masks are always recommended to protect against coronavirus, but now, with the spread of the Omicron variant, cloth masks, which were considered suitable for low-risk public settings in 2020 , are not strongly recommended because they do not offer adequate protection. It is essential to wear a medical-grade mask, at least a three-ply surgical one, to protect against this variant ${ }^{2}$.

\section{Third dose vaccination}

The third dose injection of COVID-19 vaccines has been rolled out to enhance protection and maintain immunity since the summer because of the evidence indicating reductions in infection and disease following the three-shot vaccination 1,3,4. Therefore, although there were unresolved ethical issues regarding equity in access to the vaccines ${ }^{3}$, the three-dose vaccination program is on the agenda, and so far, about 400 million third doses have been injected worldwide ${ }^{3,4}$. New laboratory results show that the Omicron has seriously challenged the potency of two doses of the Pfizer-BioNTech vaccine in preventing infection ${ }^{5}$. With the Omicron variant spreading, recommendations and planning for speeding up the program have been considered ${ }^{4}$.

\section{Updating vaccines}

Because data on the effectiveness of current vaccines for the Omicron variant were not favorable, PfizerBioNTech and Moderna are working on a specific vaccine ${ }^{1,5,6}$. Moderna could supply two to three billion vaccine doses in 2022; however, assigning all production while other variants are in circulation is a challenge ${ }^{6,7}$. Another challenge is creating the two-tier system that delivers out-of-date vaccines to developing countries 1.

\section{Pursuing universal vaccine}

According to scientific evidence and ecological reality, novel coronaviruses will continue to infect animals and be a potential threat to humans, and as a result, viruses with unknown transmissibility and lethality may appear at any time. The development of a long-lasting vaccine against all or most coronaviruses for all ages by applying the knowledge gained from characterizing the range of genetic diversity in animal species and better understanding the pathogenesis of the disease should be considered ${ }^{7}$. In addition, the fact that the Omicron variant was potentially developed from a patient with chronic COVID-19 who was immunized with a vaccine may indicate that the virus is opportunistic in its development and mutations to escape the immune system. The role of vaccine-induced immunity in generating novel variants should be considered when planning regular vaccine shots ${ }^{8}$.

\section{Concluding remark}

Wearing medical-grade masks and injecting a third dose from the current vaccines are the most available and fastest solutions to protect against the Omicron. Developing its updated specific vaccines and the universal vaccine are further scientific steps. At the same time, ethical topics and issues regarding the variants generation after vaccination should not be overlooked.

Keywords: COVID-19 Pandemic, Mask, Mass active immunization, SARS-CoV-2 


\section{Protection Against the Omicron}

Funding Statement

This paper did not receive any specific grant from funding agencies in the public, commercial, or not-for-profit sectors.

\section{Conflict of Interest}

The authors have no conflict of interest.

\section{References}

1. Burki TK. Omicron variant and booster COVID-19 vaccines. Lancet Respir Med 2021:S2213-2600(21)00559-2.

2. Michelson A. Your cloth mask is not good enough protection against Omicron, according to an expert-here's why. Business Insider; 2021. Available from: https://www.businessinsider.com/cloth-masks-are-not-good-enough-protection-against-omicron-expert2021-12. Accessed Dec 25, 2021.

3. Dolgin E. Omicron is supercharging the COVID vaccine booster debate. Nature. $2021 \mathrm{Dec} 2$.

4. Mahase E. Covid-19: Omicron and the need for boosters. BMJ 2021 Dec 14;375:n3079.

5. Steenhuysen J. Explainer: Omicron vs COVID-19 vaccines: What more do we need to know? Reuters; 2021. Available from: https://www.reuters.com/business/healthcare-pharmaceuticals/omicron-vs-covid-19-vaccines-what-more-do-we-need-know-2021-1209/. Accessed Dec 25, 2021.

6. AFP Bureaus. Moderna boss says vaccines likely no match for Omicron: FT. FRANCE 24;2021. Available from: https:// www.france24.com/en/live-news/20211130-moderna-boss-says-vaccines-likely-no-match-for-omicron-ft. Accessed Dec 25, 2021.

7. Morens DM, Taubenberger JK, Fauci AS. Universal coronavirus vaccines-an urgent need. N Engl J Med 2021 Dec 15.

8. Li X. Omicron: call for updated vaccines. J Med Virol 2021 Dec 20. 